\title{
BMJ Case Reports
}

\section{Submission template for full cases}

\section{TITLE OF CASE}

Metastatic Pancreatic Adenocarcinoma Presenting As An Occipital Haemorrhage

SUMMARY Up to 150 words summarising the case presentation and outcome (this will be freely available online)

We report a case of a 63-year-old female who presented to the emergency department with an occipital haemorrhage secondary to a pancreatic cerebral metastasis. Pancreatic cancer is the ninth most common cancer in women in Australia and distant disease is present in $70 \%$ of patients with pancreatic cancer at time of diagnosis. However, metastases to the brain are rare, accounting for only $0.33 \%-0.57 \%$ cases ante-mortem. Herein, we discuss the management of this unusual case to highlight the importance of recognizing unusual CNS involvement of cancers, a problem which may be increasing in prevalence.

\section{BACKGROUND Why you think this case is important - why did you write it up?}

Pancreatic cancer is the tenth most common cancer in men and ninth most common cancer in women in Australia, metastatic disease is present in $70 \%$ of patients with pancreatic cancer at the time of diagnosis [1]. However, spread of disease to the brain is exceedingly rare and has an incidence of $0.33 \%-0.57 \%$ in ante-mortem cases as compared to $7.9 \%$ in post-mortem diagnoses [2]. This may be due to the short life expectancy which limits time for manifestation of neurological symptoms. The median survival after cerebral involvement is less than 3 months, likely due to rapid growth of the tumour [1]. Common sites of metastases of pancreatic cancers include the peritoneum, lungs and local tumour invasion or hepatic involvement [2].

With the widespread use of gemcitabine, median survival of patients with pancreatic cancer has been prolonged and consequently, a growing number of cases of brain metastases may be seen in the future. Thus, it is vital that suspicious neurological symptoms should lead to early investigation study, and possibly treatment of cerebral metastasis [3].

\section{CASE PRESENTATION Presenting features, medical/social/family history}

A 63-year-old female presented to with retro-orbital headache and scintillating scotoma and diminished coordination. She denied any significant past surgical or medical history. She had no family history of any medical illnesses and had no previous history of cancer. Examination and investigations were otherwise normal. Magnetic resonance imaging (MRI) showed a haemorrhagic area in the left occipital lobe suggestive of an amyloid angiopathy bleed or a small haemorrhagic transformation of a stroke. She was managed initially as a haemorrhagic conversion of a stroke. (Figure 1)

The patient presented again four months later to the emergency department with recurrent right sided retro-orbital pain, visual aura, increasing visual disturbance and recurrent falls. She had also been experiencing hot sweats, night chills, a flu like illness, anorexia, nausea and vomiting. There was no significant past medical or surgical history, nor risk factors associated with malignancy.

On examination, there was obvious psychomotor slowing and right-sided neglect with agraphia without alexia and left and right agnosia. Cranial nerve examination revealed a right homonymous hemianopia and had a right facial droop. She had equal power bilaterally in her limbs. Her abdomen remained soft and non-tender. 
Lipase on admission was 29 (range $<60$ ). ALP 151 U/L (range 120-440), GGT 158 U/L (range $<22$ ), ALT $61 \mathrm{U} / \mathrm{L}$ (range 5-30), AST $66 \mathrm{U} / \mathrm{L}$ (range <38), unconjugated bilirubin 8 (range <20), conjugated bilirubin $<4$ (range $<4$ ). Full blood count was unremarkable.

Computed tomography (CT) scan performed in the emergency department showed a large haemorrhagic area in the left occipital lobe associated vasogenic oedema now causing sulcal and ventricular effacement. A low attenuation loculated region within the left occipital lobe suggested a cystic lesion possibly related to previous insult or a cystic intracranial mass.

A new MRI showed a drastic change in the appearance when compared to four months ago. There was now a $6.7 \mathrm{~cm}$ heterogenous solid cystic mass in the left occipital lobe invading the falx cerebri and the adjacent left occipital horn, with surrounding vasogenic oedema. The multiple large cystic cavities demonstrated marked haemosiderin deposition, and MRI spectroscopy suggested an aggressive lesion, likely a metastasis. (Figure 2)

Positron Emission Tomography (PET) CT Chest/Abdomen/Pelvis showed findings consistent with a primary infiltrating lesion at the uncinate process of the pancreas with resulting common bile duct and pancreatic duct obstruction and SMA occlusion, locoregional lymph nodes metastases in the small bowel mesentery and multiple bilateral pulmonary nodules. (Figure 3 )

\section{DIFFERENTIAL DIAGNOSIS If relevant}

Infective processes were initially considered as this is an atypical pattern of metastases of pancreatic cancer. However, Cryptococcal Ag, Quantiferon Gold and HIV were negative. She subsequently developed biliary obstruction and an endoscopic retrograde cholangiopancreatography (ERCP) was performed and an endoluminal stent was placed to decompress the biliary system. Pancreatic core biopsy obtained from the uncinate process showed atypical cells suspicious for malignancy. (Figure 4) Lung lesion biopsy showed no evidence of malignancy. Baseline Ca 19-9 was then done, returning a level of $2200 \mathrm{kU} / \mathrm{L}$ (range $<35$ ).

\section{TREATMENT If relevant}

A decision for cranial debulking surgery was made in a multidisciplinary team due to increasing mass effect and ongoing diagnostic uncertainty. The mass was debulked via a left craniotomy and histopathology confirmed the lesion to be consistent with metastatic carcinoma of mucinous differentiation from a pancreaticobiliary tumour. (Figure 5) (Figure 6) The patient underwent one course of adjuvant whole brain radiotherapy (WBRT), receiving 20Gy over five sessions. Patient declined palliative chemotherapy and opted for palliative symptomatic management with dexamethasone, haloperidol and analgesia.

\section{OUTCOME AND FOLLOW-UP}

She continued to deteriorate; her cognition remained intact but became increasing fatigued. She continued to decline functionally, and she passed away 9 months after initial presentation.

\section{DISCUSSION Include a very brief review of similar published cases}

In addition to pancreatic mucinous adenocarcinomas, reports of cerebral metastases from pancreatic somatostatinomas and pancreatic acinar cell tumours have also been described. Symptoms described in literature range from neurological like vertigo and sudden bilateral hearing loss (more common) to abdominal such as pneumoperitoneum and obstructive jaundice (less common)[4]. Le Cesne A et al. suggest that this subgroup of tumours form an unpredictable pattern of metastatic dissemination due to its higher propensity of early metastasis. [4] [5] 
Treatment modalities for cranial pathology include resection followed by radiation, resection alone, or WBRT [2]. Lemke et al. recommend resection of metastatic lesions of pancreatic adenocarcinomas as a treatment strategy or potentially as a cure [6]. Aim of surgery would be to reduce mass effect which is the rate-limiting factor in length of survival.

Rajappa et al. reports that the current trend in the treatment of a resectable brain metastasis in patients with stable systemic disease is to consider surgical resection followed by WBRT or surgical resection followed by stereotactic radiosurgery (SRS) as a complement to surgical resection to achieve extended survival rates. The advantage of SRS over WBRT is the reduced rate of cognitive deficits. However, in our patient with metastatic pancreatic cancer (median survival 3 to 6 months [7]), WBRT has the advantage of having a prophylactic effect in the development of recurrences [4].

\section{LEARNING POINTS/TAKE HOME MESSAGES 3 to 5 bullet points - this is a required} field

1. Cancers can present with atypical patterns of metastases and can mimic the signs and symptoms of strokes.

2. It is important to recognize that CNS involvement can occur in pancreatic cancers.

3. Early imaging studies should be performed in patients with suspicious neurological symptoms and further evaluation should be undertaken in patients with unusual scans.

4. With the advent of new chemotherapy agents, systemic disease is better controlled and as a result of this, an increasing number of intracranial metastasis may be seen in diseases which usually are associated with a shorter lifespan.

5. While currently cure is not possible, early recognition may allow for palliative resection in patients with accessible lesions to try to prolong survival.

REFERENCES Vancouver style (Was the patient involved in a clinical trial? Please reference related articles)

[1] Menis J, Fontanella C, Follador A, Fasola G, Aprile G. Brain metastases from gastrointestinal tumours: Tailoring the approach to maximize the outcome. Critical Reviews in Oncology/Hematology. 2013;85(1):32-44.

[2] Kumar A, Dagar M, Herman J, Iacobuzio-Donahue C, Laheru D. CNS Involvement in Pancreatic Adenocarcinoma: a Report of Eight Cases from the Johns Hopkins Hospital and Review of Literature. Journal of Gastrointestinal Cancer. 2014;46(1):5-8.

[3] Rajappa P, Margetis K, Wernicke G, Finter P, Cope W et al. Stereotactic Radiosurgery Plays a Critical Role in Enhancing Long-term Survival in a Patient with Pancreatic Cancer Metastatic to the Brain. Anticancer Research 33: 3899-3904 (2013)

[4] El Kamar F, Jindal K, Grossbard M, Mizrachi H, Kozuch P. Pancreatic carcinoma with brain metastases: case report and literature review. Digestive and Liver Disease. 2004;36(5):355-360.

[5] Le Cesne A, Le Chevalier T, Caille P, Cvitkovic E, Contesso G, Spielmann M, et al. Metastases from cancers of unknown primary site. Data from 302 autopsies. Presse Med 1991;20:1369-73.

[6] Lemke J, Barth T, Juchems M, Kapapa T, Bruns D et al. Long-term Survival Following Resection of Brain Metastases from Pancreatic Cancer. Anticancer Research 31: 4599-4604 (2011)

[7] Chue B. Five-Year Survival of Metastatic Pancreatic Carcinoma: A Study of Courage and Hope. Gastrointest Cancer Res. 2009 Sep-Oct; 3(5): 208-211.

\section{FIGURE/VIDEO CAPTIONS figures should NOT be embedded in this document}

Figure 1: Initial MRI T2 FLAIR

Figure 2: MRI FLAIR showing progression of lesion, and increased amount of oedema and cystic enhancement. 
Figures 3: Poorly defined, infiltrating soft tissue mass centred on the uncinate process of pancreas, displaying pathologic uptake of FDG (41mm diameter; SUVmax 9.0) consistent with a primary infiltrating lesion.

Figure 4: FNA pancreas $\mathrm{x} 40$ - clusters of large epithelial cells present showing mild to moderate atypia, some of which appear papillary and some of which are present in cohesive clusters.

Figure 5: Metastasis to brain x5: cerebral parenchyma infiltrated by metastatic adenocarcinoma. Figure 6: Metastasis to brain x10: cystically dilated glands lined by atypical columnar mucinous type epithelium with small islands and clusters of cells infiltrating the adjacent cerebral parenchyma.

\section{Copyright Statement}

I, Jessica Yan-Seen Ng, The Corresponding Author, has the right to assign on behalf of all authors and does assign on behalf of all authors, a full assignment of all intellectual property rights for all content within the submitted case report (other than as agreed with the BMJ Publishing Group Ltd) ("BMJ Group")) in any media known now or created in the future, and permits this case report (if accepted) to be published on BMJ Case Reports and to be fully exploited within the remit of the assignment as set out in the assignment which has been read. (http://casereports.bmj.com/instructions-forauthors/copyright.pdf)."

Date: $16 / 1 / 18$

PLEASE SAVE YOUR TEMPLATE WITH THE FOLLOWING FORMAT:

Corresponding author's last name and date of submission, eg,

Smith_May_2009.doc 